\title{
Variable stars in M31 from the MEGA survey
}

\author{
David R. Alves, Edward A. Baltz, Arlin Crotts, Alex Bergier, \\ Patrick Cseresnjes, Alan Gersch \\ Columbia Astrophysics Laboratory, 550 W. 120th S:., Mailcode 5247, \\ New York, NY, 10027 USA
}

\begin{abstract}
The MEGA microlensing survey is introduced and several topics about variable stars in M31 are discussed. An adopted model population of semiregular (SR) variable stars is presented, and the efficiency to recover these stars in M31 in a specific example MEGA dataset is predicted. The period-flux amplitude relation of the SRs discovered by MEGA could be used to measure the inclination of the M31 disk.
\end{abstract}

\section{Introduction}

The pioneering first generation of microlensing surveys have provided clues that both the M31 and Milky Way dark halos harbor significant numbers of massive compact objects, or MACHOs (Alcock et al. 2000; Crotts et al., these proceedings). However, no lenses have yet been proven to reside in either halo.

MEGA, which stands for Microlensing Exploration of the Galaxy and Andromeda, is a survey designed to map the spatial distribution of microlensing events in M31. If MEGA finds microlensing in the ratio of about 1 to 5 on the near and far sides of the M31 disk, respectively, the MACHO hypothesis will be vindicated. If the events are instead distributed in equal number on each side of the M31 line-of-nodes, they are more likely to be caused by star-on-star self-lensing. The latter-type events should also be more centrally concentrated than the surface brightness profile (Baltz et al. 2003). These statements assume that we know accurately the structure of M31. The significance of the result from MEGA will depend on the number of events detected, which is to say that the test of lens location is statistical. Hence one criterion for the design of the MEGA experiment is to obtain the most complete sample of microlensing possible. We are inventing techniques to efficiently analyze time-series image data that may have application to other problems in variable star research.

Variable stars play an important and synergistic role in microlensing surveys (Paczyński 1986). In particular, we can learn about galactic structure from variable stars, which provides a reference for interpreting microlensing results. For example, de Jong et al. (2003) use the variable stars from the MEGA survey to model the structure of M31 in the sense that the distributions of Miras and microlensing are compared. In addition, the databases created by microlensing surveys have had a major impact on the subject of variable stars as illustrated by many of the papers presented at this meeting (see authors Udalski, Groenewegen, Kiss, Lepischak and others). 


\section{Variable star or microlensing?}

Variable stars such as Miras and semiregular variables (SRs) can be misidentified as microlensing, particularly in M31 where most of the source stars monitored are bright red giant branch stars. It is not clear which of these two types of variables has the greater potential to masquerade as microlensing. The SRs have shorter periods, and are in principle easier to rule-out as microlensing with relatively less baseline data. However, they are 20 times more numerous than Miras and they often have irregular-amplitude pulsations. It is possible for SRs to exhibit only low-amplitude variations for many years (which might not be detected), and then undergo a single larger-amplitude variation that looks like microlensing. An example of this light curve behavior is star 7.7899.17 in the MACHO database ${ }^{1}$. Problematic Miras and SRs could be very rare and yet common enough to cause false microlensing. Although this scenario may be unlikely, it helps to illustrate why testing the microlensing hypothesis is important. The comparison of microlensing data with Miras and SRs in a colormagnitude diagram is an example of such a test. The microlensed source stars in M31 should be typically about a magnitude fainter than Miras and SRs and somewhat bluer in color (see Crotts et al., these proceedings).

\section{Optimizing variable source yield: MEGA innovations}

In order to detect microlensing a high signal-to-noise reference image is created by coadding a subset of images, and from this reference a time-series of relative flux variations is measured. The best choice for the origin of the reference is the most typical pointing in the dataset so that all of the other images overlap as much as possible. The percentage of non-overlapping area relative to any particular image $j$ in a dataset of $N$ images with dimensions $X$ and $Y$ and origins (or "pointings") $x_{j}$ and $y_{j}$ is approximately:

$$
D(j)=\sum_{i=1}^{N} \frac{\left|x_{i}-x_{j}\right|}{X}+\frac{\left|y_{i}-y_{j}\right|}{Y}
$$

Eqn. (1) is also known as the "Manhattan dissimilarity" for being the shortest distance between two points in city blocks. The image $j$ with the smallest dissimilarity $D(j)$ is the best origin for a single reference image.

Subtracting from two reference images instead of one further reduces the data loss due to telescope pointing errors. It is also possible to replace bad data in the first reference image with good data from the second image. Partitioning algorithms like Eqn. (1) can be used to pick the two best reference images (Kaufman \& Rousseeuw 1990). Double-subtraction and use of partitioning algorithms could increase MEGA source yield by about $10 \%$ (Alves et al. 2003a).

The difficulty in obtaining accurate photometry of confused sources in subtracted image data (i.e. where both positive and negative flux variations are found on spatial scales smaller than the point spread function) is another important efficiency issue. New photometry software specifically designed for crowded

${ }^{1}$ http://www.macho.mcmaster.ca/Data/MachoData.html 
sources in image subtractions could increase yields by about $10 \%$ (Alves et al. 2003b).

\section{Calibrating MEGA's yield of variable stars}

In order to calibrate detection efficiency microlensing surveys such as MEGA: (1) add fake events into the time-series image data to calibrate the source detection threshold, and (2) construct Monte Carlo models to ascertain the sensitivity of the light curve filters that are used to identify microlensing. The filter efficiency assumes a perfect noise model, while the threshold efficiency accounts for the realistic noise properties of the data. The tools for calculating microlensing detection efficiency are easily modified to accommodate variable stars like SRs and Miras (e.g., de Jong et al. 2003).

Table 1. Input SR Population and MEGA Yield Predictions

\begin{tabular}{lllllllll}
\hline $\begin{array}{l}\mathrm{P} \\
\text { day }\end{array}$ & $\mathrm{N}$ & $\begin{array}{l}\mathrm{M}_{R} \\
\mathrm{mag}\end{array}$ & $\begin{array}{l}\sigma_{R} \\
\mathrm{mag}\end{array}$ & $\begin{array}{l}\mathrm{A} \\
\mathrm{mag}\end{array}$ & $\begin{array}{l}\sigma_{A} \\
\mathrm{mag}\end{array}$ & $\mathrm{N} 1$ & $\mathrm{~N} 2$ & $\mathrm{~N} 3$ \\
\hline 25 & 309 & -3.05 & 0.25 & 0.06 & 0.01 & 280 & 38 & 2 \\
30 & 398 & -3.15 & 0.29 & 0.07 & 0.02 & 384 & 175 & 51 \\
35 & 387 & -3.22 & 0.42 & 0.07 & 0.02 & 381 & 191 & 68 \\
40 & 536 & -3.24 & 0.40 & 0.08 & 0.03 & 534 & 374 & 185 \\
45 & 493 & -3.27 & 0.39 & 0.09 & 0.03 & 493 & 424 & 263 \\
50 & 360 & -3.35 & 0.42 & 0.10 & 0.03 & 360 & 352 & 294 \\
55 & 212 & -3.34 & 0.41 & 0.10 & 0.04 & 212 & 207 & 167 \\
60 & 198 & -3.41 & 0.43 & 0.11 & 0.05 & 198 & 198 & 186 \\
65 & 176 & -3.31 & 0.59 & 0.11 & 0.05 & 176 & 176 & 146 \\
70 & 175 & -3.41 & 0.52 & 0.12 & 0.05 & 175 & 175 & 168 \\
75 & 167 & -3.39 & 0.54 & 0.13 & 0.07 & 167 & 167 & 162 \\
80 & 100 & -3.45 & 0.56 & 0.13 & 0.06 & 100 & 100 & 97 \\
85 & 107 & -3.45 & 0.67 & 0.14 & 0.06 & 107 & 107 & 105 \\
90 & 106 & -3.50 & 0.62 & 0.15 & 0.08 & 106 & 106 & 105 \\
95 & 88 & -3.37 & 0.53 & 0.15 & 0.09 & 88 & 88 & 86 \\
100 & 75 & -3.38 & 0.44 & 0.17 & 0.10 & 75 & 75 & 75 \\
\hline \hline
\end{tabular}

We have adopted a model population of SRs, which we assume to be universal, and calculated the filter efficiency to recover these stars in M31 in a specific example MEGA dataset. The reference SRs are those found in 3 square degrees of the Large Magellanic Cloud (LMC) bar in the MACHO database ${ }^{1}$. Table 1 lists the number of SRs $(\mathrm{N})$, their absolute magnitude $\left(\mathrm{M}_{R}\right.$; assuming distance and reddening corrections), and their $R$-band amplitude (A) in mag in 5-d bins of period $(P)$ for periods from 25 to $100 \mathrm{~d}$. The standard deviation of the magnitude $\left(\sigma_{R}\right)$ and amplitude $\left(\sigma_{A}\right)$ in each bin are also provided. The predicted numbers of recovered variables are listed assuming 3 values for the M31 distance: N1, N2, and N3 correspond to distance moduli of 24.05, 24.50, and $24.95 \mathrm{mag}$, respectively. These numbers do not reflect how accurately the periods can be determined; these are strictly how many we can detect. 
Recently, Paczyński \& Pindor (2000) showed that Cepheid variables do not have a universal "period-flux amplitude" relation; it depends on metallicity. Flux amplitudes are measured free from blending bias using image subtraction techniques, and thus a universal period-flux amplitude relation would be an important new tool for cosmology. The metallicity dependence of the Cepheid relation may be strong because they are young stars. In contrast SRs are a mix of many different metallicity and age stars, and they also have a periodflux amplitude relation (Jorrisen et al. 1997; Wray et al. 2003). Table 1 shows that SRs in the LMC have a well-defined period-flux amplitude relation in the $R$-band. A convincing test of the accuracy of distances derived from the SR period-flux amplitude relation would be to measure the inclination of the M31 disk. At $\pm 0.5^{\circ}$ along the tilt axis the modulus change is about $0.2 \mathrm{mag}$.

\section{Variable Stars in M31 Globular Clusters}

Globular clusters (GCs) are variable because they contain variable stars. For example, the 19 brightest variables in the Galactic GC M13 (Osborn 2000) account for $5 \%$ of the cluster's total light (18 SRs and 1 Cepheid). The typical flux variation is about $10 \%$, thus even if M13 were so far away as to be unresolved, it would be variable at the $0.5 \%$ level. If M13 were moved to the distance of M31, it would be resolved and in this case any one variable could contribute much more than $5 \%$ of the surface brightness variation depending on its location. Illustrative examples of variable stars in image subtractions of M31 GCs are presented by Alves et al. (2003b). Many types of variable star, for example period-changing Cepheids, have yet to be discovered in M31 GCs, and they could be found by MEGA. Period derivatives can be readily measured from flux-difference light curves produced by image-subtraction analysis techniques.

\section{References}

Alcock, C. et al. 2000, ApJ, 542, 281

Alves, D.R. et al. 2003a, in ESO Astrophysics Symposia, Towards an International Virtual Observatory, eds. K. Gorski \& P. Quinn (Berlin: SpringerVerlag), in press

Alves, D.R. et al. 2003b, in ASP Conf. Ser., Vol. 296, New Horizons in Globular Cluster Astronomy, eds. G. Piotto, G. Meylan, G. Djorgovski \& M. Riello, (San Francisco: ASP), in press

Baltz, E.A., Gyuk G., Crotts A. 2003, ApJ, 582, 30

de Jong, J. et al. 2003, astro-ph/0307072

Jorissen, A. et al. 1997, A\&A, 324, 578

Kaufman, L. Rousseeuw P. 1990, "Finding Groups in Data," (Wiley-Interscience, New York)

Osborn, W. 2000, AJ, 119, 2902

Paczyński, B. 1986, ApJ, 304, 1

Paczyński, B. Pindor, B. 2000, ApJ, 533, L103

Wray, J., Eyer, L., Paczyński, B. 2003, astro-ph/0310578 


\section{Discussion}

Albrow: Are you using image subtraction techniques on combinations of data from different cameras, telescopes etc?

Alves: We focus our analysis on datasets from single telescope-filter combinations. However, in some cases it is inevitable that we will subtract images from different datasets.

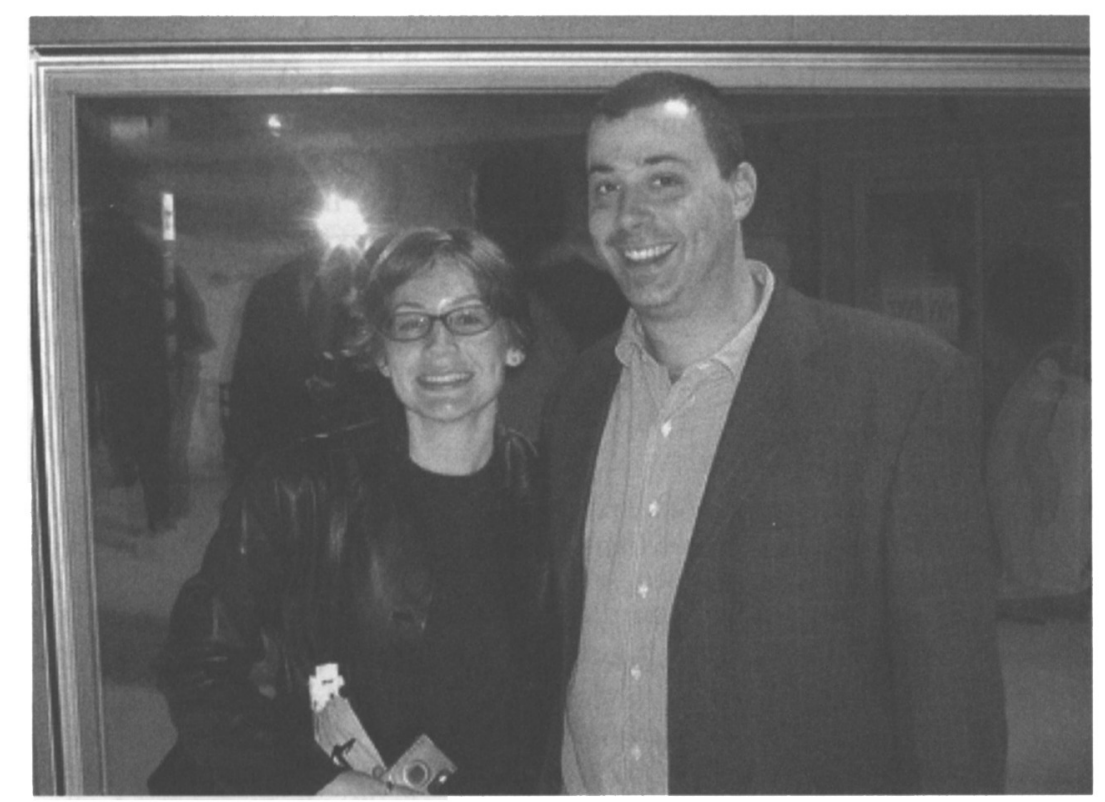

Jennifer Rados and Dave Alves 